\title{
Liquidus temperature measurements for modeling oxide glass systems relevant to nuclear waste vitrification
}

\author{
Jonathan B. Hanni, Eric Pressly, Jarrod V. Crum, Kevin B.C. Minister, Diana Tran, \\ Pavel Hrma, and John D. Vienna ${ }^{\text {a) }}$ \\ Environmental Technology Division, Pacific Northwest National Laboratory, \\ Richland, Washington 99354
}

(Received 9 March 2005; accepted 30 August 2005)

\begin{abstract}
Liquidus temperatures $\left(T_{\mathrm{L}}\right)$ were measured, and primary phases were determined for 50 (from an initial test matrix of 76) compositions within the $\mathrm{Al}_{2} \mathrm{O}_{3}-\mathrm{B}_{2} \mathrm{O}_{3}-\mathrm{CaO}-\mathrm{Na}_{2} \mathrm{O}-\mathrm{SiO}_{2}$ glass-forming system and its constituent ternary subsystems. Strong linear correlations have been found between composition and $T_{\mathrm{L}}$ for melts within the same primary phase fields. The $T_{\mathrm{L}}$ and primary phase data are being used to develop and refine a modified associate species model (ASM). The impacts of $\mathrm{Fe}_{2} \mathrm{O}_{3}, \mathrm{Li}_{2} \mathrm{O}, \mathrm{NiO}, \mathrm{ZrO}_{2}, \mathrm{Cr}_{2} \mathrm{O}_{3}, \mathrm{ZnO}$, and $\mathrm{MnO}$ additions on the $T_{\mathrm{L}}$ of two baseline glass compositions are reported. These data are intended as benchmarks for further expansion of the ASM or other silicate melt solution models of nuclear waste glasses.
\end{abstract}

\section{INTRODUCTION}

The Department of Energy (DOE) is currently constructing the world's largest nuclear waste vitrification facility to treat and immobilize nuclear wastes from the Hanford Site in southeastern Washington State. ${ }^{1}$ One of the primary concerns for operation on the electric melter in the vitrification plant is the deleterious effects of crystal accumulation in the melter. Crystals generally have higher density than the glass melt and tend to settle to the bottom of the melter where they can clog pouring spouts ${ }^{2}$ or cause electrical short circuits. ${ }^{3}$

Many of the Hanford wastes contain high concentrations of soda and alumina, and nepheline $\left(\mathrm{NaAlSiO}_{4}\right)$ may precipitate from these glasses as the melt cools. Nepheline formation during cooling may significantly decrease the chemical durability of the glass since each mole of nepheline formed removes two moles of network-forming components (one mol each of $\mathrm{Al}$ and $\mathrm{Si}$ ) for each mol of modifier $(\mathrm{Na}){ }^{2}{ }^{2}$ Constraints placed on glass composition to limit the formation of crystals in the melter or during cooling often reduce the amount of waste that can be tolerated in the glass. ${ }^{4,5}$

To better formulate nuclear waste glasses, a model capable of predicting the thermodynamics of waste glass melts is needed. With this model, one should be able to calculate the temperature at which crystals can form in multicomponent waste glass melts (the

\footnotetext{
a) Address all correspondence to this author.

e-mail: john.vienna@pnl.gov

DOI: $10.1557 / J M R .2005 .0424$
}

liquidus temperature $T_{\mathrm{L}}$ ) as a function of composition. A partnership between Oak Ridge National Laboratory, Pennsylvania State University, and Pacific Northwest National Laboratory (PNNL) has been established to develop thermodynamic models for the waste glass melt solution. A modified associate species model (ASM) is being developed. ${ }^{6-9}$ To facilitate the development of the ASM $T_{\mathrm{L}}$ data sets from glass melt systems with progressively more components are being measured.

Waste glasses are primarily composed of $\mathrm{Al}_{2} \mathrm{O}_{3}, \mathrm{~B}_{2} \mathrm{O}_{3}$, $\mathrm{Na}_{2} \mathrm{O}, \mathrm{CaO}, \mathrm{Li}_{2} \mathrm{O}, \mathrm{Fe}_{2} \mathrm{O}_{3}, \mathrm{ZrO}_{2}$, and $\mathrm{SiO}_{2} \cdot{ }^{4}$ Even relatively small concentrations of $\mathrm{ZnO}, \mathrm{Cr}_{2} \mathrm{O}_{3}, \mathrm{NiO}$, and $\mathrm{MnO}$ strongly impact the amount and composition of the crystalline phases that form in these melts as they cool. ${ }^{4,7,10-14}$ Table I summarizes the concentrations of oxides in typical Hanford waste glasses; also shown are the concentrations of oxides in typical wastes that are being vitrified at the Defense Waste Processing Facility (DWPF) at DOE's Savannah River site near Aiken, South Carolina. ${ }^{15}$ In this paper, we report the $T_{\mathrm{L}}$ and primary phases for melts made up of subsets of these 12 components. The initial testing focused on the five-component $\mathrm{Al}_{2} \mathrm{O}_{3}, \mathrm{~B}_{2} \mathrm{O}_{3}, \mathrm{Na}_{2} \mathrm{O}, \mathrm{CaO}$, and $\mathrm{SiO}_{2}$ (ABCNS) system. Additional compositions were selected from the ternary systems $\mathrm{CaO}, \mathrm{Na}_{2} \mathrm{O}$, and $\mathrm{SiO}_{2}$ and $\mathrm{CaO}, \mathrm{Al}_{2} \mathrm{O}_{3}$, and $\mathrm{SiO}_{2}$. Two baseline glasses were selected from the ABCNS glasses in this study, and varying concentrations of $\mathrm{Fe}_{2} \mathrm{O}_{3}, \mathrm{Li}_{2} \mathrm{O}, \mathrm{NiO}, \mathrm{ZrO}_{2}, \mathrm{Cr}_{2} \mathrm{O}_{3}$, $\mathrm{ZnO}$, and $\mathrm{MnO}$ were added individually to determine their effects on the $T_{\mathrm{L}}$ and primary phase formation for the baseline glass melts. 
TABLE I. Composition ranges for typical waste glass components (mass\%). ${ }^{15}$

\begin{tabular}{lcccccc}
\hline \hline & Hanford & LAW & Hanford & HLW & DWPF & HLW \\
\hline $\mathrm{Al}_{2} \mathrm{O}_{3}$ & 2 & 12 & 3 & 18 & 2.4 & 5.5 \\
$\mathrm{~B}_{2} \mathrm{O}_{3}$ & 5 & 15 & 4 & 20 & 6.8 & $11.5^{\mathrm{a}}$ \\
$\mathrm{CaO}$ & 0 & 8 & 0 & 8 & 0.7 & 1.3 \\
$\mathrm{Cr}_{2} \mathrm{O}_{3}$ & $\cdots$ & $\cdots$ & 0 & 1 & 0 & 0.2 \\
$\mathrm{Fe}_{2} \mathrm{O}_{3}$ & 0 & $8^{\mathrm{a}}$ & 1 & 18 & 8.5 & 12.4 \\
$\mathrm{~K}_{2} \mathrm{O}$ & 0 & $5^{\mathrm{a}}$ & 0.05 & 3 & 2.1 & 2.6 \\
$\mathrm{Li} \mathrm{O}_{2} \mathrm{O}$ & 0 & 6 & 0.05 & 6 & 4.3 & 5 \\
$\mathrm{MgO}$ & 0 & 4.3 & 0.05 & 3 & $0^{\mathrm{a}}$ & 2.1 \\
$\mathrm{MnO}$ & $\cdots$ & $\cdots$ & 0.02 & 10 & 1.1 & 2.8 \\
$\mathrm{Na} \mathrm{O}_{2} \mathrm{O}$ & 5 & 23 & 5 & 20 & 7.8 & $15^{\mathrm{a}}$ \\
$\mathrm{NiO}$ & $\cdots$ & $\cdots$ & 0.1 & 3 & 0.1 & 1.2 \\
$\mathrm{P}_{2} \mathrm{O}_{5}$ & 0 & 3 & 0 & 3 & $\cdots$ & $\cdots$ \\
$\mathrm{SO}_{3}$ & 0 & 1.5 & 0 & 0.5 & & \\
$\mathrm{SiO}_{2}$ & 36 & 60 & 33 & 60 & 49.1 & 55.1 \\
$\mathrm{SrO}$ & $\cdots$ & $\cdots$ & 0 & 10 & $\cdots$ & $\cdots$ \\
$\mathrm{ThO}_{2}$ & $\cdots$ & $\cdots$ & 0 & 4 & $\cdots$ & $\cdots$ \\
$\mathrm{TiO}_{2}$ & 0 & 5 & 0 & 2 & 0.2 & 0.4 \\
$\mathrm{U}_{3} \mathrm{O}_{8}$ & $\cdots$ & $\cdots$ & 0 & 12 & 0.8 & 5 \\
$\mathrm{ZnO}$ & 0 & 3 & 0 & 3 & $\cdots$ & $\cdots$ \\
$\mathrm{ZrO}_{2}$ & 0 & 6 & 0 & 10 & $\cdots$ & $\cdots$ \\
\hline \hline
\end{tabular}

${ }^{a}$ Modified ranges.

\section{DESCRIPTION OF MODIFIED ASSOCIATE SPECIES MODEL}

The ASM, originally developed by Hastie and Bonnel $^{16,17}$ and Shakhmatkin et al. ${ }^{18,19}$ has been used successfully to calculate the solution behavior of multicomponent oxide minerals and melts. In the model, the solution is represented as a mixture of associate species such as $\mathrm{NaAlO}_{2}, \mathrm{NaBO}_{2}$, and $\mathrm{Si}_{2} \mathrm{O}_{4}$. The associate species contain between one and three non-oxygen types of elements whose number must add up to 2 to provide a consistent basis for molecular size (e.g., $\mathrm{NaAlSiO}_{4}$ stoichiometry is multiplied by $2 / 3$ or $\mathrm{Na}_{0.67} \mathrm{Al}_{0.67} \mathrm{Si}_{0.67} \mathrm{O}_{2.67}$ to maintain appropriate non-oxygen content). In the basic ASM, the species in the liquid are mixed ideally to generate a melt solution. However, in the modified ASM being developed, a limited number of regular solution terms are included to allow for immiscibility in the melt. The excess enthalpy of mixing is taken into account through the energy of formation for the liquid associate species. This model has enjoyed great success in complicated oxide minerals and melts because of its simple construction and implicit tie to the type of bonding that occurs. Various species seldom would have more than two types of elements as second nearest neighbors (second to oxygen) in silicate melts. The components with the strongest mixing interactions are likely to be within the associate species. Therefore, the ideal solution is a good first approximation for mixing energies. Besmann, Spear, and colleagues ${ }^{6-9}$ give detailed discussions of the advantages of this model for glass melts and discuss its preliminary development.

\section{EXPERIMENTAL METHODS}

Liquidus temperature has been measured for seven test matrices, three containing ( $\mathrm{mol} \%): \mathrm{Al}_{2} \mathrm{O}_{3}(0.1-25)$, $\mathrm{B}_{2} \mathrm{O}_{3}(0-20), \mathrm{CaO}(0-20), \mathrm{Na}_{2} \mathrm{O}(9.1-33.3)$, and $\mathrm{SiO}_{2}$ (35.7-80); one comprised of (mol\%): $\mathrm{CaO}(22-44)$, $\mathrm{Na}_{2} \mathrm{O}$ (2.1-16), and $\mathrm{SiO}_{2}$ (51-67); one containing (mol\%) $\mathrm{CaO}$ (34-52), $\mathrm{Al}_{2} \mathrm{O}_{3}$ (1.7-11), and $\mathrm{SiO}_{2}$ (43-57); and two others, each composed of a baseline glass chosen from the initial test matrix with individual additions of the following components (mol\%): $\mathrm{Fe}_{2} \mathrm{O}_{3}(0-5), \mathrm{Li}_{2} \mathrm{O}$ (0-13), $\mathrm{NiO}(0-2), \mathrm{ZrO}_{2}(0-3), \mathrm{Cr}_{2} \mathrm{O}_{3}(0-0.25), \mathrm{ZnO}$ (0-4.5), and $\mathrm{MnO}(0-5)$.

\section{A. $\mathrm{Al}_{2} \mathrm{O}_{3}-\mathrm{B}_{2} \mathrm{O}_{3}-\mathrm{CaO}-\mathrm{Na}_{2} \mathrm{O}-\mathrm{SiO}_{2}$ glass network}

The ABCNS test matrices are presented in Table II. These compositions are all based primarily on the $\mathrm{Na}_{2} \mathrm{O}-\mathrm{Al}_{2} \mathrm{O}_{3}-\mathrm{SiO}_{2}$ ternary system (see Fig. 1). ${ }^{20}$ Glasses ABCNS-01 through -04 were chosen because they are stoichiometrically equivalent to $\mathrm{NaAlSiO}_{4}$ (nepheline), $\mathrm{NaAlSi}_{2} \mathrm{O}_{6}, \mathrm{NaAlSi}_{3} \mathrm{O}_{8}$ (albite), and $\mathrm{NaAlSi}_{4} \mathrm{O}_{10}$, respectively, although glass -04 lies within an immiscibility dome. These four compounds are well suited for comparison with both measured $T_{\mathrm{L}}$ data and ASM-predicted $T_{\mathrm{L}}$ because they are well documented in the literature. ${ }^{20}$ Glasses ABCNS-05 through -08 are based upon the first four with the addition of $\mathrm{B}_{2} \mathrm{O}_{3}$ in a $1: 1: 1$ ratio to $\mathrm{Na}_{2} \mathrm{O}$ and $\mathrm{Al}_{2} \mathrm{O}_{3}$; likewise, glasses ABCNS-09 through -12 have $\mathrm{CaO}$ in a $1: 1: 1$ ratio to $\mathrm{Na}_{2} \mathrm{O}$ and $\mathrm{Al}_{2} \mathrm{O}_{3}$, and -13 through -16 contain $\mathrm{B}_{2} \mathrm{O}_{3}$ and $\mathrm{CaO}$, both in $0.5: 1: 1$ ratios to $\mathrm{Na}_{2} \mathrm{O}$ and $\mathrm{Al}_{2} \mathrm{O}_{3}$. Glasses ABCNS-17 through -40 are statistically formulated to cover a broad range of compositions for all five components in a three compositional layer design (outer layer, inner layer, and centroid). Glass ABCNS-17 is the compositional centroid of these glass formulations, ABCNS-18 through -27 comprise an outer layer, and $\mathrm{ABCNS}-27$ through -40 are the inner layer.

Liquidus temperatures could not be measured for many of the glasses within the initial test matrix because of liquid-liquid immiscibility, inability to fabricate glass melts at $\leqslant 1600{ }^{\circ} \mathrm{C}$, or measurement difficulties; $T_{\mathrm{L}}$ was too high for the furnaces (maximum temperature $\sim 1600{ }^{\circ} \mathrm{C}$ ) or too low to be measured in laboratory time scales (e.g., equilibration time of months or years rather than days). Glasses ABCNS-41 through -48 were added to the matrix to provide additional data points. The ABCNS-2-XX glasses were also added to the matrix to generate additional $\mathrm{Al}_{2} \mathrm{O}_{3}-\mathrm{B}_{2} \mathrm{O}_{3}-\mathrm{Na}_{2} \mathrm{O}-\mathrm{SiO}_{2} T_{\mathrm{L}}$ data. Glass ABCNS-05 was re-measured with an alternate technique (melt-back, see Sec. III. E) and labeled ABCNS-05B. Melt-back was used to measure $T_{\mathrm{L}}$ for the ABCNS-2-XX glasses as well.

The ABCNS-3-XX glasses were added to measure the effects of $\mathrm{B}_{2} \mathrm{O}_{3}$ concentration near the boundaries of the 
TABLE II. $\mathrm{Al}_{2} \mathrm{O}_{3}-\mathrm{B}_{2} \mathrm{O}_{3}-\mathrm{CaO}-\mathrm{Na}_{2} \mathrm{O}-\mathrm{SiO}_{2}$ (ABCNS) glass test matrix. Compositions are given in mol fraction. $T_{\mathrm{L}}$ values have a measurement uncertainty of $\pm 5^{\circ} \mathrm{C}$.

\begin{tabular}{|c|c|c|c|c|c|c|c|}
\hline Glass ID & $\mathrm{Al}_{2} \mathrm{O}_{3}$ & $\mathrm{~B}_{2} \mathrm{O}_{3}$ & $\mathrm{CaO}$ & $\mathrm{Na}_{2} \mathrm{O}$ & $\mathrm{SiO}_{2}$ & $T_{\mathrm{L}}\left({ }^{\circ} \mathrm{C}\right)$ & Primary phase \\
\hline ABCNS-01 & 0.2500 & 0.0000 & 0.0000 & 0.2500 & 0.5000 & 1510 & Carnegieite \\
\hline ABCNS-02 & 0.1667 & 0.0000 & 0.0000 & 0.1667 & 0.6667 & 1124 & Nepheline \\
\hline ABCNS-03 & 0.1250 & 0.0000 & 0.0000 & 0.1250 & 0.7500 & 1085 & Below XRD detection limit \\
\hline ABCNS-04 & 0.1000 & 0.0000 & 0.0000 & 0.1000 & 0.8000 & $\ldots$ & $\cdots$ \\
\hline ABCNS-05 & 0.2000 & 0.2000 & 0.0000 & 0.2000 & 0.4000 & 875 & Nepheline \\
\hline ABCNS-05B & 0.2000 & 0.2000 & 0.0000 & 0.2000 & 0.4000 & 875 & Nepheline \\
\hline ABCNS-06 & 0.1429 & 0.1429 & 0.0000 & 0.1429 & 0.5714 & $\cdots$ & $\cdots$ \\
\hline ABCNS-07 & 0.1111 & 0.1111 & 0.0000 & 0.1111 & 0.6667 & $\cdots$ & $\cdots$ \\
\hline ABCNS-08 & 0.0909 & 0.0909 & 0.0000 & 0.0909 & 0.7273 & $\cdots$ & $\cdots$ \\
\hline ABCNS-09 & 0.2000 & 0.0000 & 0.2000 & 0.2000 & 0.4000 & 1303 & $\mathrm{Na}_{1.45} \mathrm{Al}_{1.45} \mathrm{Si}_{0.55} \mathrm{O}_{4}$, gehlenite \\
\hline ABCNS-10 & 0.1429 & 0.0000 & 0.1429 & 0.1429 & 0.5714 & 1133 & Nepheline \\
\hline ABCNS-11 & 0.1111 & 0.0000 & 0.1111 & 0.1111 & 0.6667 & 1190 & Pseudowollastonite \\
\hline ABCNS-12 & 0.0909 & 0.0000 & 0.0909 & 0.0909 & 0.7273 & $\cdots$ & $\cdots$ \\
\hline ABCNS-13 & 0.2000 & 0.1000 & 0.1000 & 0.2000 & 0.4000 & 1147 & Nepheline \\
\hline ABCNS-14 & 0.1429 & 0.0714 & 0.0714 & 0.1429 & 0.5714 & 955 & Andesine \\
\hline ABCNS-15 & 0.1111 & 0.0556 & 0.0556 & 0.1111 & 0.6667 & 1100 & Andesine \\
\hline ABCNS-16 & 0.0909 & 0.0455 & 0.0455 & 0.0909 & 0.7273 & $\cdots$ & $\cdots$ \\
\hline ABCNS-17 & 0.1196 & 0.0718 & 0.0771 & 0.1506 & 0.5809 & 1001 & Wollastonite \\
\hline ABCNS-18 & 0.0909 & 0.1176 & 0.1240 & 0.1068 & 0.5607 & 1001 & Wollastonite \\
\hline ABCNS-19 & 0.1712 & 0.0200 & 0.1516 & 0.2225 & 0.4347 & 1198 & Carnegieite \\
\hline ABCNS-20 & 0.1901 & 0.0000 & 0.0534 & 0.1753 & 0.5812 & $\ldots$ & $\ldots$ \\
\hline ABCNS-21 & 0.0909 & 0.0914 & 0.0271 & 0.0909 & 0.6996 & $\cdots$ & $\cdots$ \\
\hline ABCNS-22 & 0.1166 & 0.1166 & 0.1308 & 0.1946 & 0.4414 & 935 & Nepheline \\
\hline ABCNS-23 & 0.0909 & 0.0000 & 0.0171 & 0.1878 & 0.7042 & $\cdots$ & $\cdots$ \\
\hline ABCNS-24 & 0.2044 & 0.0047 & 0.0713 & 0.0987 & 0.6209 & $\cdots$ & $\cdots$ \\
\hline ABCNS-25 & 0.0909 & 0.1070 & 0.0219 & 0.0909 & 0.6893 & $\cdots$ & $\cdots$ \\
\hline ABCNS-26 & 0.1867 & 0.0000 & 0.0285 & 0.1350 & 0.6498 & $\cdots$ & $\cdots$ \\
\hline ABCNS-27 & 0.0909 & 0.1030 & 0.1086 & 0.1663 & 0.5311 & 984 & Wollastonite \\
\hline ABCNS-28 & 0.1274 & 0.0622 & 0.0500 & 0.1375 & 0.6229 & $\cdots$ & $\ldots$ \\
\hline ABCNS-29 & 0.0764 & 0.1156 & 0.0915 & 0.1375 & 0.5790 & $\cdots$ & $\cdots$ \\
\hline ABCNS-30 & 0.1014 & 0.1005 & 0.0912 & 0.1375 & 0.5694 & 984 & Wollastonite \\
\hline ABCNS-31 & 0.0953 & 0.0782 & 0.1045 & 0.2105 & 0.5115 & 935 & Wollastonite \\
\hline ABCNS-32 & 0.1037 & 0.0542 & 0.0921 & 0.1394 & 0.6105 & 1061 & Wollastonite \\
\hline ABCNS-33 & 0.1442 & 0.0500 & 0.0740 & 0.1727 & 0.5591 & 1001 & Nepheline/wollastonite \\
\hline ABCNS-34 & 0.1594 & 0.0830 & 0.0661 & 0.1435 & 0.5480 & 1052 & Anorthite/albite \\
\hline ABCNS-35 & 0.0883 & 0.0819 & 0.0792 & 0.1927 & 0.5578 & 915 & Wollastonite \\
\hline ABCNS-36 & 0.1126 & 0.0987 & 0.0982 & 0.1563 & 0.5342 & 984 & Nepheline \\
\hline ABCNS-37 & 0.1431 & 0.1021 & 0.0746 & 0.1491 & 0.5312 & 905 & Anorthite/albite \\
\hline ABCNS-38 & 0.0639 & 0.1275 & 0.1035 & 0.1892 & 0.5158 & 886 & Wollastonite \\
\hline ABCNS-39 & 0.1367 & 0.0644 & 0.0753 & 0.1804 & 0.5432 & 974 & Nepheline \\
\hline ABCNS-40 & 0.1125 & 0.1156 & 0.0911 & 0.1484 & 0.5324 & 956 & Wollastonite \\
\hline ABCNS-41 & 0.1429 & 0.0807 & 0.0889 & 0.2119 & 0.4757 & 1044 & Nepheline \\
\hline ABCNS-42 & 0.2418 & 0.2000 & 0.0000 & 0.1582 & 0.4000 & $\cdots$ & Corundum \\
\hline ABCNS-43 & 0.0142 & 0.0383 & 0.0830 & 0.2054 & 0.6591 & 841 & $\mathrm{Na}_{2} \mathrm{Ca}_{3} \mathrm{Si}_{6} \mathrm{O}_{16}$ \\
\hline ABCNS-44 & 0.1653 & 0.0895 & 0.0953 & 0.1005 & 0.5494 & 1198 & Anorthite/albite \\
\hline ABCNS-45 & 0.0455 & 0.0460 & 0.0587 & 0.2315 & 0.6183 & 814 & Combeite \\
\hline ABCNS-46 & 0.2331 & 0.1424 & 0.0000 & 0.1999 & 0.4246 & $\cdots$ & Corundum \\
\hline ABCNS-47 & 0.0006 & 0.1272 & 0.0067 & 0.1312 & 0.7343 & 1080 & Cristobalite/tridymite \\
\hline ABCNS-48 & 0.1486 & 0.0735 & 0.0988 & 0.1000 & 0.5792 & 1167 & Anorthite/albite \\
\hline ABCNS-2-01 & 0.2222 & 0.1111 & 0.0000 & 0.2222 & 0.4444 & 1178 & Nepheline \\
\hline ABCNS-2-02 & 0.1538 & 0.0769 & 0.0000 & 0.1538 & 0.6154 & $\cdots$ & $\cdots$ \\
\hline ABCNS-2-03 & 0.1176 & 0.0588 & 0.0000 & 0.1176 & 0.7059 & $\cdots$ & $\cdots$ \\
\hline ABCNS-2-04 & 0.0952 & 0.0476 & 0.0000 & 0.0952 & 0.7619 & $\cdots$ & $\cdots$ \\
\hline ABCNS-3-01 & 0.1234 & 0.0502 & 0.0000 & 0.3498 & 0.4766 & 990 & Nepheline \\
\hline ABCNS-3-01b & 0.1234 & 0.0502 & 0.0000 & 0.3498 & 0.4766 & 990 & Nepheline \\
\hline ABCNS-3-02 & 0.1740 & 0.0515 & 0.0000 & 0.1275 & 0.6470 & 1409 & Mullite/corundum \\
\hline ABCNS-3-03 & 0.0842 & 0.0489 & 0.0000 & 0.3485 & 0.5184 & 830 & Nepheline \\
\hline ABCNS-3-04 & 0.1920 & 0.0521 & 0.0000 & 0.1375 & 0.6184 & 1347 & Mullite/corundum \\
\hline ABCNS-3-05 & 0.1079 & 0.1693 & 0.0000 & 0.3059 & 0.4169 & 830 & Nepheline \\
\hline ABCNS-3-06 & 0.0739 & 0.1656 & 0.0000 & $\begin{array}{c}0.3057 \\
\text { (continued) }\end{array}$ & 0.4548 & 718 & Nepheline \\
\hline
\end{tabular}


TABLE II. $\mathrm{Al}_{2} \mathrm{O}_{3}-\mathrm{B}_{2} \mathrm{O}_{3}-\mathrm{CaO}-\mathrm{Na}_{2} \mathrm{O}-\mathrm{SiO}_{2}$ (ABCNS) glass test matrix. Compositions are given in mole fraction. $T_{\mathrm{L}}$ values have a measurement uncertainty of $\pm 5^{\circ} \mathrm{C}$. (continued)

\begin{tabular}{cccccccc}
\hline \hline Glass ID & $\mathrm{Al}_{2} \mathrm{O}_{3}$ & $\mathrm{~B}_{2} \mathrm{O}_{3}$ & $\mathrm{CaO}$ & $\mathrm{Na}_{2} \mathrm{O}$ & $\mathrm{SiO}_{2}$ & $T_{\mathrm{L}}\left({ }^{\circ} \mathrm{C}\right)$ & Primary phase \\
\hline ABCNS-3-07 & 0.1517 & 0.1731 & 0.0000 & 0.1111 & 0.5641 & 1290 & Mullite/aluminum borate \\
ABCNS-3-08 & 0.1671 & 0.1748 & 0.0000 & 0.1197 & 0.5383 & 1315 & Mullite/aluminum borate \\
ABCNS-4-01 & 0.0000 & 0.0000 & 0.4442 & 0.0413 & 0.5145 & $\ldots$ & $\ldots$ \\
ABCNS-4-02 & 0.0000 & 0.0000 & 0.4072 & 0.0207 & 0.5721 & $\ldots$ & $\ldots$ \\
ABCNS-4-03 & 0.0000 & 0.0000 & 0.4055 & 0.0684 & 0.526 & $\ldots$ & $\ldots$ \\
ABCNS-4-04 & 0.0000 & 0.0000 & 0.3441 & 0.0456 & 0.6103 & $\ldots$ & $\ldots$ \\
ABCNS-4-05 & 0.0000 & 0.0000 & 0.3344 & 0.1147 & 0.5509 & $\ldots$ & $\ldots$ \\
ABCNS-4-06 & 0.0000 & 0.0000 & 0.2553 & 0.0777 & 0.6671 & 1298 & Pseudowollastonite \\
ABCNS-4-07 & 0.0000 & 0.0000 & 0.2417 & 0.1617 & 0.5966 & 1193 & Pseudowollastonite \\
ABCNS-4-08 & 0.0000 & 0.0000 & 0.2185 & 0.122 & 0.6596 & 1208 & Pseudowollastonite \\
ABCNS-5-01 & 0.0413 & 0.0000 & 0.4442 & 0.0000 & 0.5145 & $\ldots$ & $\ldots$ \\
ABCNS-5-02 & 0.0207 & 0.0000 & 0.4072 & 0.0000 & 0.5721 & $\ldots$ & $\ldots$ \\
ABCNS-5-03 & 0.0684 & 0.0000 & 0.4055 & 0.0000 & 0.526 & $\ldots$ & $\ldots$ \\
ABCNS-5-04 & 0.0456 & 0.0000 & 0.3441 & 0.0000 & 0.6103 & 1393 & Pseudowollastonite \\
ABCNS-5-05 & 0.1147 & 0.0000 & 0.3344 & 0.0000 & 0.5509 & 1378 & Pseudowollastonite \\
ABCNS-5-06 & 0.0777 & 0.0000 & 0.2553 & 0.0000 & 0.6671 & 1378 & Pseudowollastonite \\
ABCNS-5-07 & 0.1617 & 0.0000 & 0.2417 & 0.0000 & 0.5966 & 1278 & Pseudowollastonite \\
ABCNS-5-08 & 0.122 & 0.0000 & 0.2185 & 0.0000 & 0.6596 & 1288 & Pseudowollastonite \\
\hline \hline
\end{tabular}

nepheline phase field of the $\mathrm{Na}_{2} \mathrm{O}-\mathrm{Al}_{2} \mathrm{O}_{3}-\mathrm{SiO}_{2}$ ternary group. Four base compositions of $\mathrm{Na}_{2} \mathrm{O}, \mathrm{Al}_{2} \mathrm{O}_{3}$, and $\mathrm{SiO}_{2}$ were measured containing 5 and $15 \mathrm{wt} \% \mathrm{~B}_{2} \mathrm{O}_{3}$ (roughly 5 and $15 \mathrm{~mol} \%$ ). Glasses ABCNS-01/-05 and -03/-06 are both on the high sodium end of the nepheline phase field; ABCNS-02/-07 and -04/-08 are in the corundum field on the high alumina side of nepheline. Glass ABCNS-3-01 showed excessive volatility during melting, so a second batch was prepared and melted at a lower temperature (labeled ABCNS-3-01b) to determine whether the volatilization had impacted the composition enough to affect $T_{\mathrm{L}}$

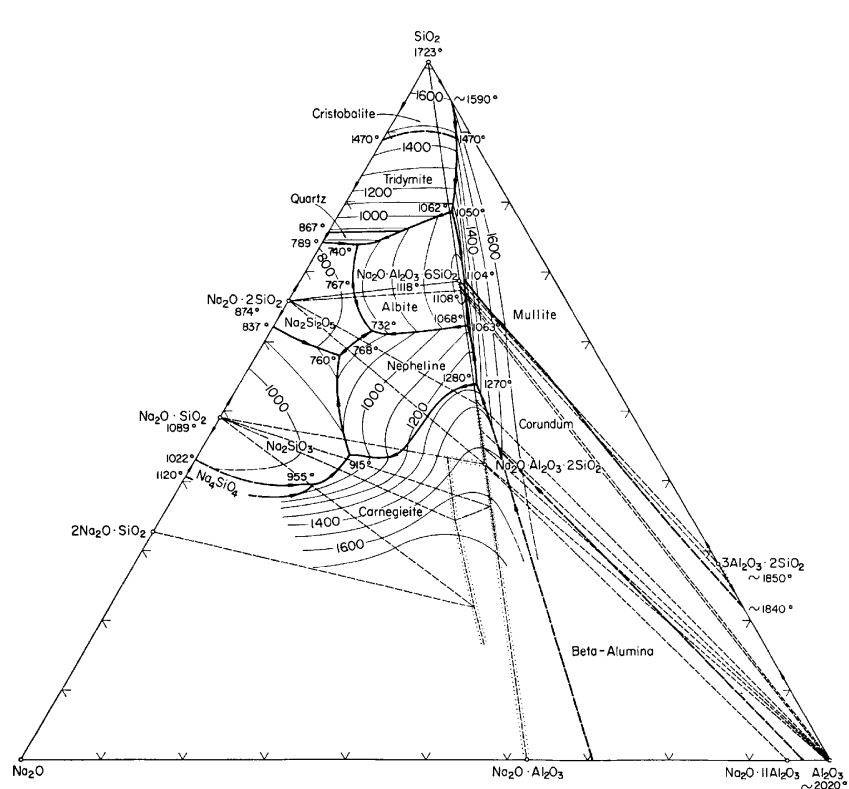

FIG. 1. Phase diagram for the $\mathrm{Na}_{2} \mathrm{O}-\mathrm{Al}_{2} \mathrm{O}_{3}-\mathrm{SiO}_{2}$ ternary oxide system. Reprinted with permission of the American Ceramic Society, www.ceramics.org. Copyright 1964. All rights reserved. ${ }^{20}$

\section{B. $\mathrm{CaO}-\mathrm{Na}_{2} \mathrm{O}-\mathrm{SiO}_{2}$ and $\mathrm{CaO}-\mathrm{Al}_{2} \mathrm{O}_{3}-\mathrm{SiO}_{2}$ ternary systems}

In addition to the ABCNS matrix, which is based primarily on the $\mathrm{Na}_{2} \mathrm{O}-\mathrm{Al}_{2} \mathrm{O}_{3}-\mathrm{SiO}_{2}$ system, two test matrices were developed for measuring $T_{\mathrm{L}}$ for glass compositions within the $\mathrm{CaO}-\mathrm{Na}_{2} \mathrm{O}-\mathrm{SiO}_{2}$ and $\mathrm{CaO}-\mathrm{Al}_{2} \mathrm{O}_{3}-$ $\mathrm{SiO}_{2}$ ternary groups. ${ }^{21,22}$ The $\mathrm{CaO}-\mathrm{Na}_{2} \mathrm{O}-\mathrm{SiO}_{2}$ glasses have been designated ABCNS-4-01 through ABCNS-408 and are marked on the ternary phase diagram given in Fig. 2. The $\mathrm{CaO}-\mathrm{Al}_{2} \mathrm{O}_{3}-\mathrm{SiO}_{2}$ glasses are listed as

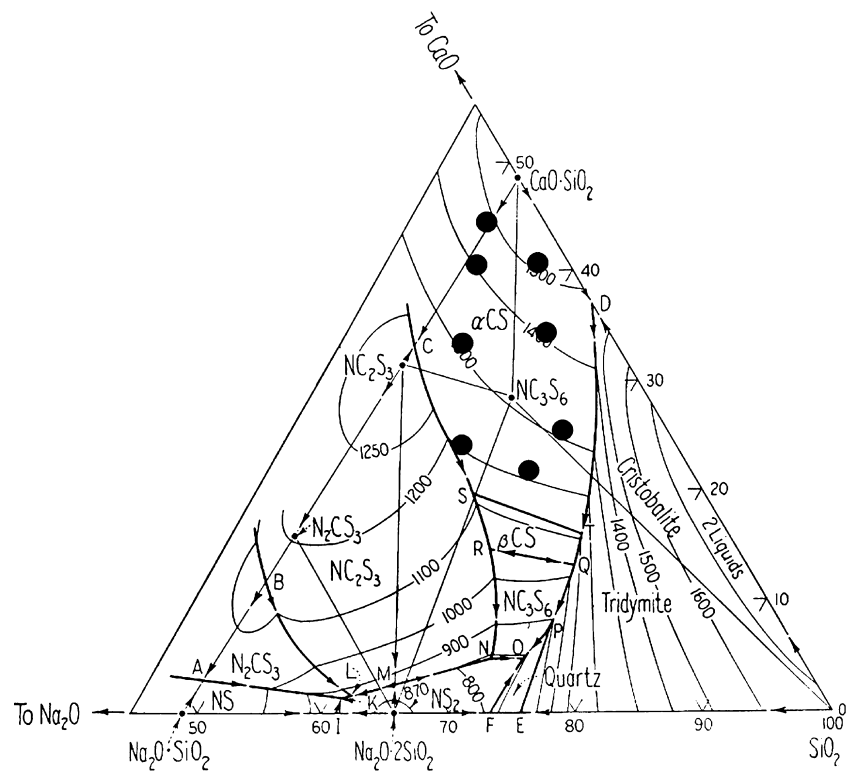

FIG. 2. Phase diagram for the $\mathrm{CaO}-\mathrm{Na}_{2} \mathrm{O}-\mathrm{SiO}_{2}$ ternary oxide system. Black circles indicate the compositions of the ABCNS-4-XX glasses. Reprinted with permission of the American Ceramic Society, www. ceramics.org. Copyright 1964. All rights reserved. ${ }^{20}$ 
ABCNS-5-01 through ABCNS-5-08 and marked on the diagram presented in Fig. 3. Compositions for both of these test matrices are also found in Table II.

\section{Expanded oxide matrices}

Two of the ABCNS glasses were selected as baseline glasses for the expanded oxide matrix. Glass ABCNS-13 was selected because it is stoichiometrically equivalent to nepheline, the most common primary phase in the ABCNS matrix; it is designated ABCNS-6-00 in the new matrix. Glass ABCNS-27, now designated ABCNS-7-00, was chosen because it represents the melts well in the wollastonite $\left(\mathrm{CaSiO}_{3}\right)$ primary phase field, the second most common phase field in the ABCNS study. The oxides $\mathrm{Fe}_{2} \mathrm{O}_{3}, \mathrm{Li}_{2} \mathrm{O}, \mathrm{NiO}, \mathrm{ZrO}_{2}, \mathrm{Cr}_{2} \mathrm{O}_{3}, \mathrm{NiO}$, and $\mathrm{MnO}$ were added individually to each baseline glass at two or more concentrations by diluting the baseline components in equal relative proportion. Table III presents the ABCNS-6-XX and ABCNS-7-XX test matrices, respectively.

\section{Sample fabrication}

Glasses were batched at the target compositions listed in Tables II and III with reagent grade chemicals (brand, minimum purity, and particle size information are listed in Table IV); component concentrations were estimated to have $<0.05 \%$ relative uncertainty (except for $\mathrm{Cr}_{2} \mathrm{O}_{3}$, which was present in such small quantities that relative uncertainty is estimated to be $<0.16 \%$ ). Each batch was

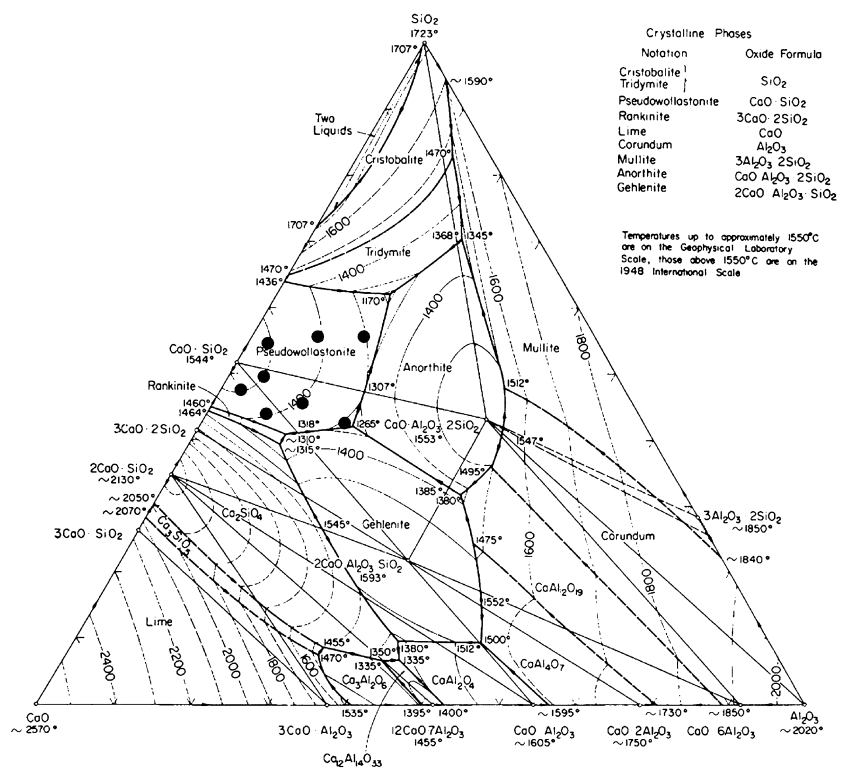

FIG. 3. Phase diagram for the $\mathrm{CaO}-\mathrm{Al}_{2} \mathrm{O}_{3}-\mathrm{SiO}_{2}$ ternary oxide system. Black circles indicate the compositions of the ABCNS-5-XX glasses. Reprinted with permission of the American Ceramic Society, www. ceramics.org. Copyright 1964. All rights reserved. ${ }^{22}$ mixed for $5 \mathrm{~min}$ in an agate mill and melted for $1 \mathrm{~h}$ in $\mathrm{Pt} /(10 \%-\mathrm{Rh})$ crucibles with lids (some with high melting temperatures, such as ABCNS-3-02, were melted for $2 \mathrm{~h}$ ). A tungsten-carbide mill was used to grind the glasses for $5 \mathrm{~min}$ before melting for a second time to ensure homogeneity. Melts were quenched on a stainless steel plate. The quenched glasses were crushed and sieved between \#5 and \#40 mesh screens to obtain particle sizes between $4 \mathrm{~mm}$ and $425 \mu \mathrm{m}$. The crushed glass was soaked in ethanol, ultrasonically cleaned for $2 \mathrm{~min}$, and dried overnight at $100{ }^{\circ} \mathrm{C}$ to evaporate the ethanol.

\section{E. $T_{L}$ measurement}

Three measurement techniques were used to determine $T_{\mathrm{L}}$ in this study. For the ABCNS glasses, crushed glasses were placed in $\mathrm{Pt} /(10 \% \mathrm{Rh})$ boats $30 \mathrm{~cm}$ long by $0.7 \mathrm{~cm}$ wide and $1.4 \mathrm{~cm}$ deep. These were placed inside a linear gradient temperature furnace $\left(1{ }^{\circ} \mathrm{C} / \mathrm{mm}\right)$ for $24 \mathrm{~h}$, and $T_{\mathrm{L}}$ was determined by locating the point along the long axis of the boat where crystals were undetectable with transmitted light optical microscopy at magnifications of up to $500 \times{ }^{23}$ This method worked well to narrow down $T_{\mathrm{L}}$ to an approximate temperature, but isothermal heat treatments were used to bound $T_{\mathrm{L}}$ with much greater precision. Crushed samples were placed in $1 \mathrm{~cm}^{3} \mathrm{Pt} /(10 \%-\mathrm{Rh})$ crucibles with tight-fitting lids and heat treated at specific temperatures for sufficient time to reach equilibrium (24-96 h). Melt samples were rapidly quenched in air after heat treatment. Optical microscopy was used to determine the presence of crystals in the quenched samples. Measurements continued at different temperatures until $T_{\mathrm{L}}$ was bounded within a $10{ }^{\circ} \mathrm{C}$ interval; crystals were present at one temperature and not present at $10{ }^{\circ} \mathrm{C}$ higher.

A technique known as melt-back was used to measure $T_{\mathrm{L}}$ for ABCNS-05B and the ABCNS-2-XX glasses. Crushed glass samples were heat treated at temperatures below $T_{\mathrm{L}}$ in $1 \mathrm{~cm}^{3} \mathrm{Pt} / \mathrm{Rh}$ crucibles to promote crystallization. The samples were then heat treated at successively higher temperatures until all crystals had dissolved in the melt.

Scanning-electron microscopy with energy dispersive spectroscopy (SEM-EDS) and x-ray diffraction (XRD) were used to identify primary crystalline phases for the glasses. Generally, samples that had been heat treated within $50{ }^{\circ} \mathrm{C}$ of $T_{\mathrm{L}}$ were ground into fine powder for XRD analysis; samples that had been heat treated at lower temperatures were used if the higher temperature samples contained insufficient crystalline phase to give a good XRD pattern. SEM was used to determine the presence of crystals that were difficult to see with optical microscopy, to determine the composition of crystals formed, and to confirm the compositions of selected glasses. 
TABLE III. ABCNS-6-XX and ABCNS-7-XX glass matrices (compositions given in mol percent). Oxide concentrations were added to the ABCNS-6-00 (same composition as ABCNS-13 in Table II) and ABCNS-7-00 (same composition as ABCNS-27 in Table II) baseline glasses.

\begin{tabular}{|c|c|c|c|c|c|c|c|c|c|}
\hline ABCNS- & $\mathrm{Fe}_{2} \mathrm{O}_{3}$ & $\mathrm{Li}_{2} \mathrm{O}$ & $\mathrm{NiO}$ & $\mathrm{ZrO}_{2}$ & $\mathrm{Cr}_{2} \mathrm{O}_{3}$ & $\mathrm{ZnO}$ & $\mathrm{MnO}$ & $T_{\mathrm{L}}\left({ }^{\circ} \mathrm{C}\right)$ & Primary phase \\
\hline $6-00$ & $\ldots$ & $\ldots$ & $\ldots$ & $\ldots$ & $\cdots$ & $\cdots$ & $\cdots$ & 1158 & Nepheline \\
\hline $6-01$ & 4.61 & $\cdots$ & $\ldots$ & $\ldots$ & $\ldots$ & $\ldots$ & $\ldots$ & 1155 & Nepheline/hematite \\
\hline $6-02$ & 2.24 & $\ldots$ & $\cdots$ & $\ldots$ & $\cdots$ & $\ldots$ & $\cdots$ & 1145 & Nepheline \\
\hline $6-03$ & $\ldots$ & 12.91 & $\ldots$ & $\ldots$ & $\ldots$ & $\ldots$ & $\ldots$ & 996 & Nepheline \\
\hline 6-04 & $\ldots$ & 6.70 & $\ldots$ & $\ldots$ & $\cdots$ & $\ldots$ & $\ldots$ & 1075 & Nepheline \\
\hline $6-05$ & $\cdots$ & $\cdots$ & 1.86 & $\cdots$ & $\cdots$ & $\cdots$ & $\cdots$ & 1155 & $\mathrm{NiO} /$ nepheline \\
\hline $6-06$ & $\cdots$ & $\cdots$ & 0.93 & $\cdots$ & $\cdots$ & $\cdots$ & $\cdots$ & 1145 & Nepheline \\
\hline $6-07$ & $\cdots$ & $\cdots$ & $\cdots$ & 2.88 & $\cdots$ & $\ldots$ & $\ldots$ & 1585 & Baddeleyite \\
\hline $6-08$ & $\cdots$ & $\cdots$ & $\cdots$ & 1.42 & $\cdots$ & $\cdots$ & $\cdots$ & 1235 & Baddeleyite \\
\hline $6-09$ & $\cdots$ & $\cdots$ & $\cdots$ & $\cdots$ & 0.23 & $\cdots$ & $\cdots$ & 1245 & Nepheline \\
\hline $6-10$ & $\ldots$ & $\cdots$ & $\cdots$ & $\ldots$ & 0.11 & $\ldots$ & $\ldots$ & 1215 & Nepheline \\
\hline $6-11$ & $\cdots$ & $\cdots$ & $\cdots$ & $\cdots$ & $\cdots$ & 4.29 & $\cdots$ & 1205 & Nepheline \\
\hline $6-12$ & $\ldots$ & $\ldots$ & $\cdots$ & $\ldots$ & $\cdots$ & 2.14 & $\cdots$ & 1155 & Nepheline \\
\hline $6-13$ & $\ldots$ & $\ldots$ & $\ldots$ & $\ldots$ & $\ldots$ & $\ldots$ & 4.90 & 1135 & Nepheline \\
\hline $6-14$ & $\ldots$ & $\ldots$ & $\ldots$ & $\ldots$ & $\ldots$ & $\ldots$ & 2.45 & 1155 & Nepheline \\
\hline $6-15$ & $\cdots$ & $\cdots$ & $\cdots$ & 2.15 & $\cdots$ & $\cdots$ & $\cdots$ & 1485 & Baddeleyite \\
\hline $7-00$ & $\cdots$ & $\cdots$ & $\cdots$ & $\cdots$ & $\cdots$ & $\cdots$ & $\cdots$ & 967 & Wollastonite \\
\hline $7-01$ & 4.31 & $\cdots$ & $\cdots$ & $\cdots$ & $\cdots$ & $\cdots$ & $\cdots$ & 940 & Wollastonite \\
\hline $7-02$ & 2.09 & $\ldots$ & $\ldots$ & $\ldots$ & $\ldots$ & $\cdots$ & $\cdots$ & 960 & Parawollastonite \\
\hline $7-03$ & $\cdots$ & 12.15 & $\ldots$ & $\ldots$ & $\ldots$ & $\ldots$ & $\ldots$ & 940 & Wollastonite \\
\hline $7-04$ & $\cdots$ & 6.28 & $\cdots$ & $\cdots$ & $\cdots$ & $\cdots$ & $\cdots$ & 875 & Wollastonite \\
\hline $7-05$ & $\cdots$ & $\ldots$ & 1.74 & $\cdots$ & $\cdots$ & $\cdots$ & $\cdots$ & 960 & Parawollastonite \\
\hline $7-06$ & $\cdots$ & $\ldots$ & 0.87 & $\ldots$ & $\cdots$ & $\ldots$ & $\ldots$ & 1015 & Wollastonite \\
\hline $7-07$ & $\cdots$ & $\cdots$ & $\cdots$ & 2.69 & $\cdots$ & $\cdots$ & $\cdots$ & 995 & Parawollastonite \\
\hline $7-08$ & $\ldots$ & $\ldots$ & $\ldots$ & 1.33 & $\ldots$ & $\ldots$ & $\ldots$ & 985 & Wollastonite \\
\hline 7-09 & $\ldots$ & $\ldots$ & $\ldots$ & $\ldots$ & 0.21 & $\ldots$ & $\ldots$ & 1125 & Nepheline \\
\hline $7-10$ & $\ldots$ & $\ldots$ & $\ldots$ & $\ldots$ & 0.11 & $\ldots$ & $\ldots$ & 995 & Wollastonite \\
\hline $7-11$ & $\cdots$ & $\cdots$ & $\cdots$ & $\cdots$ & $\cdots$ & 4.02 & $\cdots$ & 1015 & Wollastonite \\
\hline $7-12$ & & $\cdots$ & $\cdots$ & $\cdots$ & $\cdots$ & 2.00 & $\cdots$ & 1015 & Wollastonite \\
\hline $7-13$ & $\ldots$ & $\ldots$ & $\ldots$ & $\ldots$ & $\cdots$ & $\ldots$ & 4.58 & 995 & Wollastonite \\
\hline $7-14$ & $\cdots$ & $\ldots$ & $\cdots$ & $\ldots$ & $\cdots$ & $\cdots$ & 2.29 & 1025 & Wollastonite \\
\hline $7-15$ & $\cdots$ & 9.27 & $\cdots$ & $\ldots$ & $\cdots$ & $\cdots$ & $\ldots$ & 885 & Wollastonite \\
\hline $7-16$ & $\ldots$ & 3.19 & $\ldots$ & $\ldots$ & $\ldots$ & $\ldots$ & $\ldots$ & 925 & Wollastonite \\
\hline $7-17$ & $\cdots$ & $\cdots$ & 1.30 & $\cdots$ & $\cdots$ & $\cdots$ & $\cdots$ & 965 & Wollastonite \\
\hline $7-18$ & $\ldots$ & $\ldots$ & 0.43 & $\ldots$ & $\ldots$ & $\ldots$ & $\ldots$ & 1005 & Wollastonite \\
\hline
\end{tabular}

TABLE IV. Desired oxides, chemicals used for batching, chemical brand, minimum chemical purity mass $\%$, and particle size.

\begin{tabular}{lllll}
\hline \hline Oxide & Chemical & \multicolumn{1}{c}{ Brand } & Purity $(\%)$ & Particle size $^{\text {a }}$ \\
\hline $\mathrm{Al}_{2} \mathrm{O}_{3}$ & $\mathrm{Al}_{2} \mathrm{O}_{3}$ & Fisher & 99 & \\
$\mathrm{~B}_{2} \mathrm{O}_{3}$ & $\mathrm{H}_{3} \mathrm{BO}_{3}$ & Noah & 99.8 & Granular \\
$\mathrm{CaO}$ & $\mathrm{CaCO}_{3}$ & Fisher & 99 & \\
$\mathrm{Na}_{2} \mathrm{O}$ & $\mathrm{Na}_{2} \mathrm{CO}_{3}$ & Fisher & 99.5 & \\
$\mathrm{SiO}_{2}$ & $\mathrm{SiO}_{2}$ & Fisher & 99 & -240 mesh \\
$\mathrm{Fe}_{2} \mathrm{O}_{3}$ & $\mathrm{Fe}_{2} \mathrm{O}_{3}$ & Fisher & 99.999 & -199 mesh \\
$\mathrm{Li}_{2} \mathrm{O}$ & $\mathrm{Li}_{2} \mathrm{CO}_{3}$ & Alfa Aesar & 99 & \\
$\mathrm{NiO}$ & $\mathrm{NiO}_{\mathrm{ZrO}}$ & GFS & 99 & \\
$\mathrm{ZrO}_{2}$ & Noah & 99 & -325 mesh \\
$\mathrm{Cr}_{2} \mathrm{O}_{3}$ & $\mathrm{Cr}_{2} \mathrm{O}_{3}$ & Alfa Aesar & 98 & -325 mesh \\
$\mathrm{ZnO}$ & $\mathrm{ZnO}$ & Noah & 99.9 & -200 mesh \\
$\mathrm{MnO}$ & $\mathrm{MnO}$ & Alfa Aesar & 99.99 & +200 mesh \\
\hline \hline
\end{tabular}

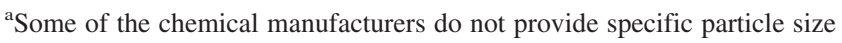
data. All chemicals used are powders.

\section{IV. $T_{L}$ AND PRIMARY PHASE MEASUREMENT RESULTS}

\section{A. $\mathrm{Al}_{2} \mathrm{O}_{3}-\mathrm{B}_{2} \mathrm{O}_{3}-\mathrm{CaO}-\mathrm{Na}_{2} \mathrm{O}-\mathrm{SiO}_{2}$ system}

Table II displays $T_{\mathrm{L}}$ and primary phase results for the ABCNS-XX, ABCNS-2-XX, and ABCNS-3-XX glasses. The $T_{\mathrm{L}}$ for glasses ABCNS-04, $-08,-12,-16$, and -29 were all unable to be measured because of multiple immiscible phases upon fabrication. Liquidus temperature could not be measured for glasses ABCNS-06, -07, ABCNS-2-02, -03, and -04 as the crystallization kinetics were too slow for the techniques used. Glasses ABCNS$20,-21,-23$ through -26 , and -28 did not completely melt at the highest operating temperature of the furnaces used $\left(\sim 1600{ }^{\circ} \mathrm{C}\right)$. When glasses ABCNS-42 and -46 were melted at $1550{ }^{\circ} \mathrm{C}$ and poured, no crystals were present; after a 24 -h heat treatment at $1550{ }^{\circ} \mathrm{C}$, both were highly 
crystalline (crystal fraction greater than 50\%). From the XRD patterns, both glasses contained corundum $\left(\mathrm{Al}_{2} \mathrm{O}_{3}\right)$, but $T_{\mathrm{L}}$ was higher than the operating temperature of the furnaces. Diffraction peaks consistent with aluminum borate were also found in the XRD pattern from glass ABCNS-42, and shorter heat treatments of 1,2 , and $4 \mathrm{~h}$ were performed to determine whether volatilization of sodium borate at $1550{ }^{\circ} \mathrm{C}$ could have caused $T_{\mathrm{L}}$ to increase. Loss of $\mathrm{Na}_{2} \mathrm{O}$ from glass increases the $T_{\mathrm{L}}$ of melts in most primary phase fields, including corundum. Crystals were found on bubbles and on the surface of the samples from shorter heat treatments, so $T_{\mathrm{L}}$ could not be determined.

\section{B. $\mathrm{CaO}-\mathrm{Na}_{2} \mathrm{O}-\mathrm{SiO}_{2}$ and $\mathrm{CaO}-\mathrm{Al}_{2} \mathrm{O}_{3}-\mathrm{SiO}_{2}$ ternary systems}

Table II also contains $T_{\mathrm{L}}$ and primary phase information for the ABCNS-4-XX and ABCNS-5-XX glasses. Glasses ABCNS-4-01 through -05 and ACBNS-5-01 through -03 all crystallized rapidly during quenching from 1500 and $1600{ }^{\circ} \mathrm{C}$; attempts to rapidly quench the melt by placing the crucible in a water bath instead of pouring the melt onto a stainless steel plate were unsuccessful in preventing crystallization. Hence, $T_{\mathrm{L}}$ was not measured for these compositions.

\section{C. $\mathrm{Cr}_{2} \mathrm{O}_{3}-\mathrm{Fe}_{2} \mathrm{O}_{3}-\mathrm{Li}_{2} \mathrm{O}-\mathrm{NiO}-\mathrm{MnO}-\mathrm{ZnO}-$ $\mathrm{ZrO}_{2}$ additions}

Primary phase and $T_{\mathrm{L}}$ results for the ABCNS-6-XX and ABCNS-7-XX glass matrices are presented in Table III. Figure 4 shows $T_{\mathrm{L}}$ as a function of oxide concentration for each component added to the ABCNS-600 and ABCNS-7-00 baseline glasses. Each oxide was added to the baseline glasses at evenly spaced concentrations. The addition of $\mathrm{ZrO}_{2}$ to the ABCNS-6-00 baseline glass had a profound impact on $T_{\mathrm{L}}$; it increased by an average of $140{ }^{\circ} \mathrm{C}$ per mol\% $\mathrm{ZrO}_{2}$. An additional glass (ABCNS-7-15) was fabricated with $2.15 \mathrm{~mol} \% \mathrm{ZrO}_{2}$ to better determine the shape of the increasing $T_{\mathrm{L}}$ curve. Additions of $\mathrm{Li}_{2} \mathrm{O}$ and $\mathrm{NiO}$ to the ABCNS-7-00 glass yielded both increasing and decreasing $T_{\mathrm{L}}$ values. Two additional glasses with intermediate concentrations of these oxides were fabricated to determine the shape and rate of change of $T_{\mathrm{L}}$ with increasing oxide concentration.

\section{DISCUSSION}

\section{A. Comparison with literature values}

Well-known literature values are available for direct comparison with ABCNS-01 through -04, ABCNS-4$\mathrm{XX}$, and ABCNS-5-XX glasses (see Figs. 1-3 and Table V). For ABCNS-01 through -03, the $T_{\mathrm{L}}$ data match the literature values reasonably well with a maximum divergence of $33{ }^{\circ} \mathrm{C} ; T_{\mathrm{L}}$ for ABCNS-04 was indeterminate because of liquid-liquid immiscibility. The observed primary phases for glasses ABCNS-01 and -02 were in agreement with carnegieite and nepheline, as shown in the phase diagram (Fig. 1). The primary phase for ABCNS-03 was undetectable in the XRD patterns after heat treatments $50{ }^{\circ} \mathrm{C}$ below $T_{\mathrm{L}}$.

The ABCNS-4-XX and ABCNS-5-XX glasses were selected such that their compositions all corresponded to $T_{\mathrm{L}}$ isotherms within the pseudowollastonite phase fields of their respective ternary groups. The glasses for which $T_{\mathrm{L}}$ and primary phase could be determined show very strong agreement with the literature values (all of these glasses contain pseudowollastonite, and the largest deviation between $T_{\mathrm{L}}$ values is $\sim 25^{\circ} \mathrm{C}$ ).

\section{B. Component concentration effects}

Empirical modeling of complex oxide melts often requires nonlinear terms. Backman et al. ${ }^{24}$ have successfully used polynomial regressive fits; Dreyfus and Dreyfus ${ }^{25}$ have used neural networks to model $T_{\mathrm{L}}$. Empirical models are not the primary goal of this work, but a firstorder fit has been used to demonstrate that, when glasses are grouped into their specific primary phase fields, composition effects can be adequately described; these component effects can then be compared to those estimated with the ASM. Regression fits to the nepheline/ carnegiete- and wollastonite/pseudowollastonite-phase field glasses include enough data to be statistically significant. An unweighted multiple linear regression of the following form was used

$$
T_{\mathrm{L}}=\sum_{i=1}^{N} a_{\mathrm{i}} x_{\mathrm{i}},
$$

where $N$ is the total number of components, and $a_{\mathrm{i}}$ and $x_{\mathrm{i}}$ are the first-order coefficient and mole fraction of the $i^{\text {th }}$ component, respectively. This form of first-order model has been used successfully to model $T_{\mathrm{L}}$ for manycomponent simulated waste glasses within the nepheline phase field. ${ }^{26}$

For the nepheline-phase field glasses, including the 2 from which carnegieite precipitated, $a_{\mathrm{i}}$ values were calculated for all 17 glasses. Glasses ABCNS-3-01 and ABCNS-3-01b were both included in this fit, since both glasses were fabricated and $T_{\mathrm{L}}$ was measured separately. The $T_{\mathrm{L}}$ for ABCNS-05 was measured multiple times with both isothermal and meltback techniques, with both techniques yielding the same value of $T_{\mathrm{L}}$. Since the same batch was used for all of these measurements, this composition was included only once in the fit. The original ABCNS-XX matrix contained 11 wollastonite-forming $\left(\mathrm{CaSiO}_{3}\right)$ glasses, including the one pseudowollastonitephase field glass and the one glass that precipitated both 

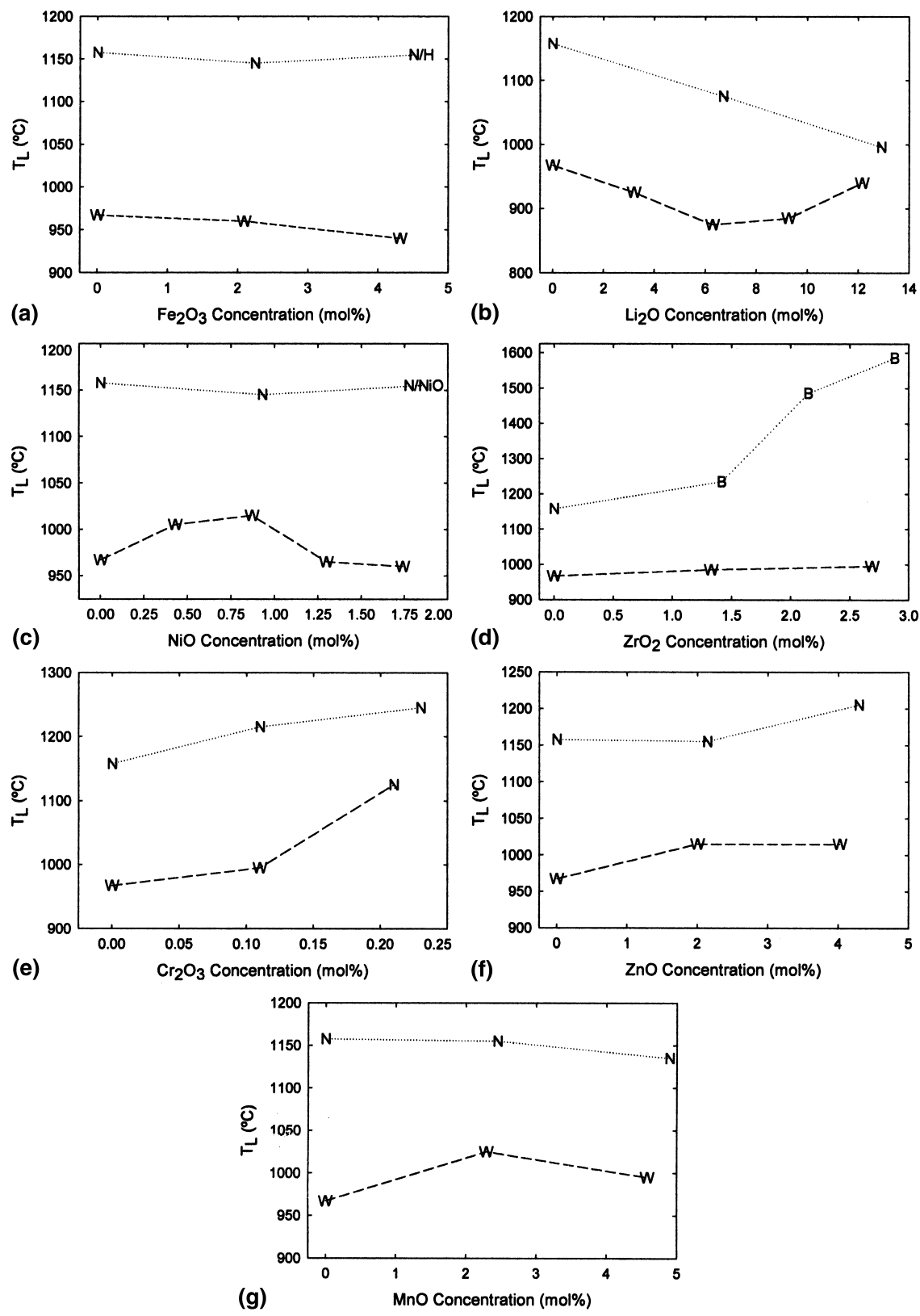

FIG. 4. $T_{\mathrm{L}}$ as a function of mol\% of oxide for ABCNS-6-XX (connected with dashed lines) and ABCNS-7-XX glasses (connected with dotted lines). Oxide additions are shown as follows: (a) $\mathrm{Fe}_{2} \mathrm{O}_{3}$, (b) $\mathrm{Li}_{2} \mathrm{O}$, (c) $\mathrm{NiO}$, (d) $\mathrm{ZrO}_{2}$, (e) $\mathrm{Cr}_{2} \mathrm{O}_{3}$, (f) $\mathrm{ZnO}$, and (g) MnO. Phases are given as $\mathrm{N}=$ nepheline $\left(\mathrm{NaAlSiO}_{4}\right), \mathrm{W}=$ wollastonite $\left(\mathrm{CaSiO}_{3}\right), \mathrm{B}=$ baddeleyite $\left(\mathrm{ZrO}_{2}\right), \mathrm{H}=$ hematite $\left(\mathrm{Fe}_{2} \mathrm{O}_{3}\right)$, and $\mathrm{NiO}=$ nickel oxide $(\mathrm{NiO})$.

nepheline and wollastonite. Equation (1) was used to fit these 11 glasses combined with the eight pseudowollastoniteforming glasses from the ABCNS-4-XX and ABCNS-5XX matrices. Table VI contains $T_{\mathrm{L}}$ coefficients $\left(a_{\mathrm{i}}\right.$ values) from both regression calculations.

The linear fits to the data sets from the nepheline- and wollastonite-phase field glasses have large $R^{2}$ values; the 17-point linear fit to the nepheline-phase field glasses has an $R^{2}$ of 0.924 , and $R^{2}$ for 19-point wollastonite/ pseudowollastonite-phase field glasses is 0.981 . These regression results suggest that a very strong first-order correlation exists between $T_{\mathrm{L}}$ and glass composition when compositions are within the same phase field. Furthermore, the phase transition between wollastonite and pseudowollastonite is linear with respect to $T_{\mathrm{L}}$.

Plots of calculated $T_{\mathrm{L}}$ versus measured $T_{\mathrm{L}}$ are shown in Fig. 5 for the 17-point nepheline model; the 19-point wollastonite model is displayed in Fig. 6. Error bars indicate $95 \%$ confidence intervals (U) and have been calculated as follows

$$
\mathrm{U}=t_{(1-\alpha), n-p} s \sqrt{\mathbf{x}\left(\mathbf{x}^{\mathrm{T}} \mathbf{X}\right)^{-1} \mathbf{x}^{\mathrm{T}}}
$$


TABLE V. Comparison of ABCNS glasses to literature data (see Figs. 1-3 and associated references).

\begin{tabular}{lllll}
\hline \hline & \multicolumn{2}{c}{ Experiment } & \multicolumn{2}{c}{ Literature } \\
\cline { 2 - 5 } Glass ID & $T_{\mathrm{L}}\left({ }^{\circ} \mathrm{C}\right)$ & Primary phase & $T_{\mathrm{L}}\left({ }^{\circ} \mathrm{C}\right)$ & Primary phase \\
\hline ABCNS-01 & $1510 \pm 5$ & Carnegieite & $1525^{19}$ & $1150^{19}$ \\
ABCNS-02 & $1124 \pm 5$ & Nepheline & $1118^{19}$ & Carnegieite \\
ABCNS-03 & $1085 \pm 5$ & Pseudowollastonite & Albite \\
ABCNS-4-06 & $1298 \pm 5$ & Pseudowollastonite & $\sim 1300^{20}$ & Pseudowollastonite \\
ABCNS-4-07 & $1193 \pm 5$ & Pseudowollastonite & $\sim 1200^{20}$ & Pseudowollastonite \\
ABCNS-4-08 & $1208 \pm 5$ & Pseudowollastonite & $\sim 1200^{20}$ & Pseudowollastonite \\
ABCNS-5-04 & $1393 \pm 5$ & Pseudowollastonite & $\sim 1400^{21}$ & Pseudowollastonite \\
ABCNS-5-05 & $1378 \pm 5$ & Pseudowollastonite & $\sim 1400^{21}$ & Pseudowollastonite \\
ABCNS-5-06 & $1378 \pm 5$ & Pseudowollastonite & $\sim 1300^{21}$ & Pseudowollastonite \\
ABCNS-5-07 & $1278 \pm 5$ & Pseudowollastonite & Pseudowollastonite \\
ABCNS-5-08 & $1288 \pm 5$ & $1300^{21}$ & Pseudowollastonite \\
\hline \hline
\end{tabular}

TABLE VI. $T_{\mathrm{L}}$ coefficients $a_{\mathrm{i}}$ [Eq. (1)] for the nepheline and wollastonite linear fits.

\begin{tabular}{lcc}
\hline \hline Oxide & $\begin{array}{c}\text { Nepheline } \\
(17 \text { points })\end{array}$ & $\begin{array}{c}\text { Wollastonite } \\
(19 \text { points })\end{array}$ \\
\hline $\mathrm{Al}_{2} \mathrm{O}_{3}$ & 3542 & 723 \\
$\mathrm{~B}_{2} \mathrm{O}_{3}$ & -814 & -189 \\
$\mathrm{CaO}$ & 1158 & 1429 \\
$\mathrm{Na}_{2} \mathrm{O}$ & 934 & 147 \\
$\mathrm{SiO}_{2}$ & 589 & 1369 \\
\hline \hline
\end{tabular}

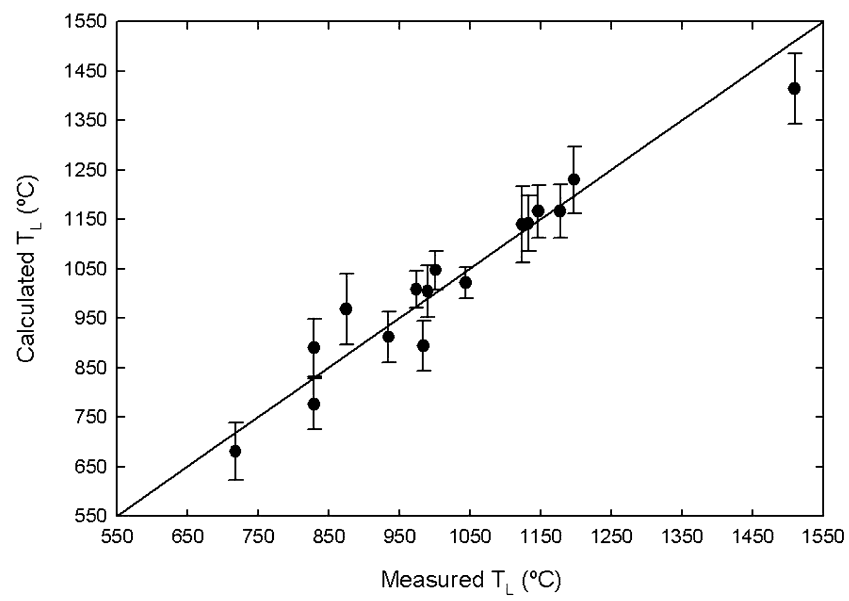

FIG. 5. Comparison of calculated $T_{\mathrm{L}}$ versus measured $T_{\mathrm{L}}$ corresponding to a 17-point fit for glasses that precipitate nepheline (15 glasses) and carnegieite (2 glasses).

where $t$ is the student's $t$-score corresponding to $1-\alpha=$ confidence limit, $n$ is the total number of samples, $p$ is the number of predictors (components), $s$ is the standard error, $\mathbf{x}$ is the composition vector for an individual glass, and $\mathbf{X}$ is the composition matrix for all glasses in the model.

\section{Expanded oxide matrix data}

Overall, the $T_{\mathrm{L}}$ for the ABCNS-7-XX glasses (wollastonite primary phase) changes less after adding the seven

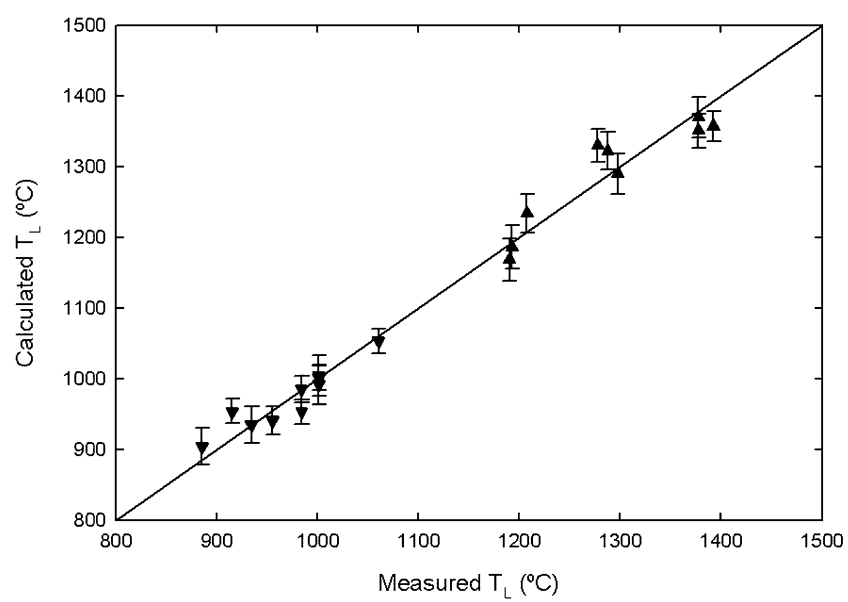

FIG. 6. Comparison of calculated $T_{\mathrm{L}}$ versus measured $T_{\mathrm{L}}$ corresponding to a 19-point fit for glasses that precipitate wollastonite (downtriangles) and pseudowollastonite (up-triangles).

oxides than did the $T_{\mathrm{L}}$ for the ABCNS-6-XX series of glasses (nepheline primary phase). In this baseline glass (ABCNS-7-00), XRD analysis indicated that only the $0.21 \mathrm{~mol} \% \mathrm{Cr}_{2} \mathrm{O}_{3}$ addition changed the primary phase from wollastonite to nepheline. The primary phase in the ABCNS-6-00 baseline glass was changed with the addition of the following: $\mathrm{ZrO}_{2}$, precipitating baddeleyite $\left(\mathrm{ZrO}_{2}\right)$ at three $\mathrm{ZrO}_{2}$ concentrations; $\mathrm{Fe}_{2} \mathrm{O}_{3}$, precipitating nepheline at all concentrations of $\mathrm{Fe}_{2} \mathrm{O}_{3}$ and hematite $\left(\mathrm{Fe}_{2} \mathrm{O}_{3}\right)$ plus nepheline at $4.61 \mathrm{~mol} \% \mathrm{Fe}_{2} \mathrm{O}_{3}$; and $\mathrm{NiO}$, precipitating nepheline at all concentrations and nickel oxide $(\mathrm{NiO})$ plus nepheline at $1.86 \mathrm{~mol} \% \mathrm{NiO}$.

With the exceptions of $\mathrm{Li}_{2} \mathrm{O}$ and $\mathrm{ZrO}_{2}$, the $T_{\mathrm{L}}$ for the ABCNS-6-XX (nepheline-precipitating) glasses was affected by the additional oxides less than the ABCNS-7$\mathrm{XX}$ glasses. The phase change from nepheline to baddeleyite accounts for the sharp increase in $T_{\mathrm{L}}$ for the ABCNS-6-XX glasses containing $\mathrm{ZrO}_{2}$. A similar $T_{\mathrm{L}}$ trend is caused by the phase change from wollastonite to nepheline in the ABCNS-7-XX glasses that contain $\mathrm{Cr}_{2} \mathrm{O}_{3}$. It is not yet clear why such a small concentration 
of $\mathrm{Cr}_{2} \mathrm{O}_{3}$ should change the phase field of ABCNS-7-XX from wollastonite to nepheline.

The data ABCNS-7-XX glasses show some intriguing effects of $\mathrm{Li}_{2} \mathrm{O}$ or $\mathrm{NiO}$ additions on $T_{\mathrm{L}}$. For $\mathrm{NiO}, T_{\mathrm{L}}$ increases sharply with up to $0.43 \mathrm{~mol} \%$, levels off until $0.87 \mathrm{~mol} \%$, and then decreases sharply to the same value as the baseline glass at $1.3 \mathrm{~mol} \% \mathrm{NiO}$, followed by a slight decrease when $\mathrm{NiO}$ reaches $1.74 \mathrm{~mol} \%$. From the XRD patterns, the NiO-bearing ABCNS-7-XX glasses precipitate wollastonite as the primary phase. However, it is possible that an additional phase is present in the sample but was not detected with the XRD technique used.

Crystals in a sample of ABCNS-7-05 that had been heat-treated at $900{ }^{\circ} \mathrm{C}$ were analyzed with SEM/EDS to

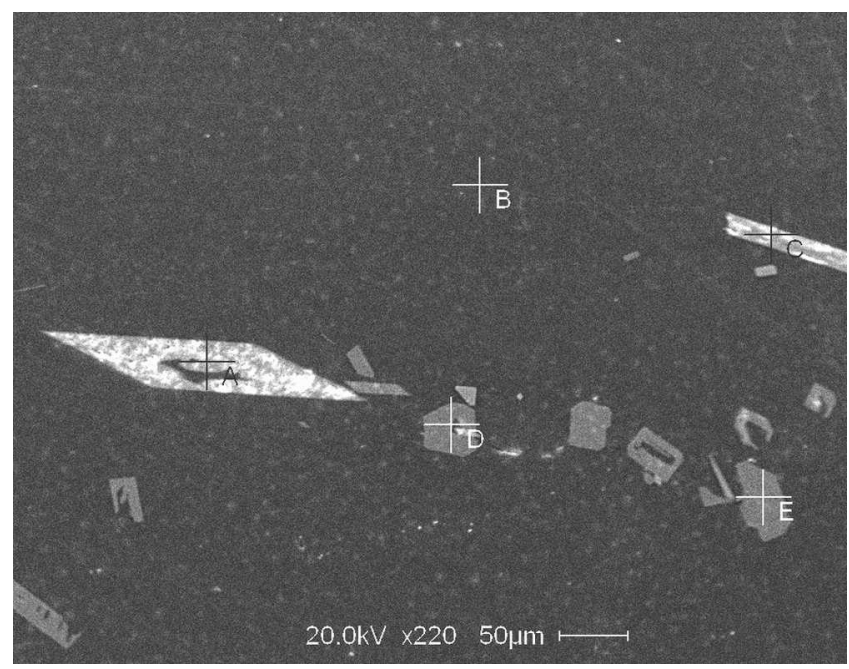

FIG. 7. SEM micrograph of ABCNS-6-05 after heat treatment at $900{ }^{\circ} \mathrm{C}$. Crosshairs indicate locations of EDS spot-scans; these data are given in Table VII.

TABLE VII. EDS results for composition ( $\mathrm{mol} \%$ ) and calculated stoichiometries for the crystals found in ABCNS-7-05 (Fig. 7).

\begin{tabular}{ccccccc}
\hline \hline Crystal & $\mathrm{Ca}$ & $\mathrm{Ni}$ & $\mathrm{Al}$ & $\mathrm{Si}$ & $\mathrm{O}$ & \multicolumn{1}{c}{ Stoichiometry } \\
\hline $\mathrm{A}$ & 26.03 & $\cdots$ & 0.73 & 25.41 & 47.83 & $\mathrm{Ca}_{1.30} \mathrm{Al}_{0.04} \mathrm{Si}_{1.27} \mathrm{O}_{2.39}$ \\
$\mathrm{C}$ & 12.32 & 11.53 & 1.43 & 24.2 & 50.52 & $\mathrm{Ca}_{1.23} \mathrm{Ni}_{1.15} \mathrm{Al}_{0.14} \mathrm{Si}_{2.42} \mathrm{O}_{5.05}$ \\
$\mathrm{D}$ & 20.01 & 1.24 & 2.56 & 25.32 & 50.87 & $\mathrm{Ca}_{1.00} \mathrm{Ni}_{0.06} \mathrm{Al}_{0.13} \mathrm{Si}_{1.27} \mathrm{O}_{2.54}$ \\
$\mathrm{E}$ & 26.5 & $\cdots$ & $\cdots$ & 24.51 & 48.99 & $\mathrm{Ca}_{1.33} \mathrm{Si}_{1.23} \mathrm{O}_{2.45}$ \\
\hline \hline
\end{tabular}

determine if a second phase was present with wollastonite. A SEM micrograph is presented in Fig. 7; the crosshairs in the photo indicate the locations of each EDS spot scan. The mol\% of each element and the calculated crystal stoichiometry for crystals A, C, D, and E (see Fig. 7) are given in Table VII. It should be noted that the EDS procedure used is qualitative rather than quantitative. ${ }^{27}$ By comparing the ratios of $\mathrm{Ca}, \mathrm{Ni}$, and $\mathrm{Si}$, crystals $\mathrm{A}, \mathrm{D}$, and $\mathrm{E}$ are consistent with $\mathrm{CaSiO}_{3}$ (wollastonite), and crystal $\mathrm{C}$ is consistent with $\mathrm{CaNiSi}_{2} \mathrm{O}_{6}$ (niopside ${ }^{28}$ ).

Location B in Fig. 7 is the bulk glass, and its composition was analyzed to compare with the target composition of ABCNS-7-05 listed in Table III. To compare the EDS determined and target glass compositions, the EDS results have been renormalized by assuming the glass contains exactly the target concentration of $\mathrm{B}_{2} \mathrm{O}_{3}$ since boron cannot be measured using the EDS technique used. Table VIII contains the actual and target compositions of ABCNS-7-05.

The ABCNS-7-XX $T_{\mathrm{L}}$ curve for $\mathrm{Li}_{2} \mathrm{O}$ appears to have a smoother response than that for $\mathrm{NiO}$. A local minimum occurs at approximately $6.28 \mathrm{~mol} \%$, corresponding to a $T_{\mathrm{L}}$ decrease of $90{ }^{\circ} \mathrm{C}$ from the $T_{\mathrm{L}}$ of the baseline glass. The ABCNS-6-XX $\mathrm{Li}_{2} \mathrm{O}$ curve has a nearly identical $90{ }^{\circ} \mathrm{C}$ drop in $T_{\mathrm{L}}$ up to this concentration. The ABCNS6 -XX curve continues to decrease in a nearly linear curve between $6.7 \mathrm{~mol} \%$ and $12.91 \mathrm{~mol} \%$, ending approximately $160{ }^{\circ} \mathrm{C}$ lower than the baseline, but the ABCNS-7-XX curve slowly increases $10{ }^{\circ} \mathrm{C}$ between $6.28 \mathrm{~mol} \%$ and $9.27 \mathrm{~mol} \%$ before sharply climbing $55^{\circ} \mathrm{C}$ by $12.15 \mathrm{~mol} \%$.

\section{CONCLUSIONS}

The primary goal of this work is to provide $T_{\mathrm{L}}$ and primary phase data for a broad range of glass melt compositions to develop and refine the ASM and/or other thermodynamic models for the prediction of waste glass melt phase behavior. To that end, this paper reports the $T_{\mathrm{L}}$ 's and primary phases of 51 glass melts in the $\mathrm{Al}_{2} \mathrm{O}_{3}-$ $\mathrm{B}_{2} \mathrm{O}_{3}-\mathrm{CaO}-\mathrm{Na}_{2} \mathrm{O}-\mathrm{SiO}_{2}$ system and 35 glass melts each containing one of the following additional components: $\mathrm{Cr}_{2} \mathrm{O}_{3}, \mathrm{Fe}_{2} \mathrm{O}_{3}, \mathrm{Li}_{2} \mathrm{O}, \mathrm{MnO}, \mathrm{NiO}, \mathrm{ZnO}$, and $\mathrm{ZrO}_{2}$.

The data presented in this paper indicate a close agreement with literature values, where literature values are

TABLE VIII. Target and mean measured (EDS) bulk glass composition of ABCNS-7-05. Ten independent measurements were averaged together to obtain the EDS composition results; uncertainties represent two standard deviations. For the EDS-measured composition, it was assumed that $\mathrm{B}_{2} \mathrm{O}_{3}$ content in the glass matched that of the target composition.

\begin{tabular}{lcccccc}
\hline \hline & $\begin{array}{c}\mathrm{B}_{2} \mathrm{O}_{3} \\
(\mathrm{~mol} \%)\end{array}$ & $\begin{array}{c}\mathrm{Al}_{2} \mathrm{O}_{3} \\
(\mathrm{~mol} \%)\end{array}$ & $\begin{array}{c}\mathrm{CaO} \\
(\mathrm{mol} \%)\end{array}$ & $\begin{array}{c}\mathrm{Na}_{2} \mathrm{O} \\
(\mathrm{mol} \%)\end{array}$ & $\begin{array}{c}\mathrm{SiO}_{2} \\
(\mathrm{~mol} \%)\end{array}$ & $\begin{array}{c}\mathrm{NiO}^{2} \\
(\mathrm{~mol} \%)\end{array}$ \\
\hline EDS & $\ldots$ & $9.13 \pm 0.50$ & $11.34 \pm 0.92$ & $11.25 \pm 2.7$ & $55.89 \pm 2.1$ & $2.27 \pm 0.30$ \\
Target & 10.12 & 8.93 & 10.68 & 16.34 & 52.19 & 1.74 \\
\hline \hline
\end{tabular}


available. This agreement is important as a means of measurement validation. Most of the compositions presented here contain more than three components, so they provide a means of examining how each subsystem of ternary inputs to the ASM or other melt thermodynamic models interact as more components are added; with these data, fit parameters can be adjusted.

Strong linear trends between component concentrations, and $T_{\mathrm{L}}$ values within the nepheline/carnegieite and wollastonite/pseudowollastonite phase fields were found. Similar (linear) trends were found previously in more complex (e.g., 20+ component) waste glass melts from which spinel, nepheline, and zirconia containing phases precipitated. . $^{2,10-14}$

\section{ACKNOWLEDGMENTS}

We would like to thank Dean Moore of PNNL for his help in performing XRD analyses. Mike Schweiger of PNNL contributed his extensive experience with laboratory procedures and measurement techniques to discussions regarding this project. Ted Besmann, Karl Spear, and Nagraj Kulkarni worked diligently to develop and refine the ASM, and they suggested many of the glass compositions studied in this work. Jonathan Hanni, Eric Pressly, and Kevin Minister would like to thank the Office of Fellowship Programs at PNNL for the opportunity to conduct this research project while holding undergraduate and graduate student fellowships at the laboratory. Denis Strachan and Wayne Cosby performed thorough reviews of this document. This work was supported by the U.S. Department of Energy Office of Science through the Environmental Management Science Program grant. Pacific Northwest National Laboratory is operated for the U.S. Department of Energy by Battelle under Contract No. DE-AC05-76RL01830.

\section{REFERENCES}

1. Design Construction, and Commissioning of the Hanford Tank Waste Treatment and Immobilization Plant. U.S. Department of Energy Contract No. DE-AC27-01RV14136, (2000, as amended).

2. D-S. Kim and P. Hrma: Models for liquidus temperature of nuclear waste glasses. Ceram. Trans. 45, 327 (1994).

3. I.G. Choi, D.F. Bickford, and J.T. Carter: Thermal effects of electrically conductive deposits in a joule-heated melter. Ceram. Trans. 29, 645 (1993).

4. D-S. Kim and J.D. Vienna: Influence of glass property restrictions on Hanford HLW glass volume. Ceram. Trans. 132, 105 (2002).

5. P. Hrma, J.D. Vienna, and M.J. Schweiger: Liquidus temperature limited waste loading maximization for vitrified HLW. Ceram. Trans. 72, 449 (1996).

6. T.M. Besmann and K.E. Spear: Thermochemical modeling of oxide glasses. J. Am. Ceram. Soc. 85, 2887 (2002).

7. T.M. Besmann, K.E. Spear, and J.D. Vienna: Extension of the modified associate species thermochemical model for high-level nuclear waste: Inclusion of chromia, in Scientific Basis for
Nuclear Waste Management XXVI, edited by R.J. Finch and D.B. Bullen (Mater. Res. Soc. Symp. Proc. 757, Warrendale, PA, 2003), p. 195.

8. T.M. Besmann, N.S. Kulkarni, and K.E. Spear: Thermochemical and phase equilibria property prediction for oxide glass systems based on the modified associate species approach, in High Temperature Corrosion and Materials Technology IV, edited by E. Opila, P. Hou, T. Maruyama, B. Pieraggi, M. McNallan, D. Shifler, and E. Wuchina. (The Electrochemical Society, Pennington, NJ, 2004), p. 557.

9. K.E. Spear, T.M. Besmann, and E.E. Beahm: Thermochemical modeling of glass: Application to high-level nuclear waste glass. MRS Bull. 24(4), 37 (1999).

10. J.V. Crum, P. Hrma, M.J. Schweiger, and G.F. Piepel: Liquidus temperature models for high-level waste glasses that precipitate zirconium-containing crystalline phases. Ceram. Trans. 87, 271 (1998).

11. T. Plaisted, P. Hrma, J. Vienna, and A. Jiricka: Liquidus temperature and primary phase in high-zirconia high-level waste borosilicate glasses, in Scientific Basis for Nucelar Waste Management XXIII, edited by R.W. Smith and D.W. Shoesmith (Mater. Res. Soc. Symp. Proc. 608, Warrendale, PA, 2000), p. 709.

12. J.D. Vienna, P. Hrma, J.V. Crum, and M. Mika: Liquidus temperature-composition model for multi-component glasses in the $\mathrm{Fe}, \mathrm{Cr}, \mathrm{Ni}$, and $\mathrm{Mn}$ spinel primary phase field. J. Non-Cryst. Solids 292, 1 (2001).

13. P. Hrma, J. Vienna, J. Crum, and G. Piepel: Liquidus temperature of high-level waste borosilicate glasses with spinel primary phase, in Scientific Basis for Nucelar Waste Management XXIII, edited by R.W. Smith and D.W. Shoesmith (Mater. Res. Soc. Symp. Proc. 608, Warrendale, PA, 2000), p. 671.

14. M. Mika, M.J. Schweiger, J.D. Vienna, and P. Hrma: Liquidus temperature of spinel precipitating high-level waste glasses, in Scientific Basis for Nucelar Waste Management XX, edited by W.J. Gray and I.R. Tridy (Mater. Res. Soc. Symp. Proc., 465, Pittsburgh, PA, 1997), p. 71.

15. J.D. Vienna: Nuclear waste glasses, in Properties of GlassForming Melts, edited by L.D. Pye, I. Joseph, and A. Montenero (CRC Press, Boca Raton, FL, 2005). pp. 391-403.

16. J.W. Hastie and D.W. Bonnell: A predictive phase-equilibrium model for multicomponent oxide mixtures. 2. Oxides of $\mathrm{Na}-\mathrm{K}-$ Ca-Mg-Al-Si. High Temp. Sci. 19, 275 (1985).

17. D.W. Bonnell and J.W. Hastie: A predictive thermodynamic model for complex high-temperature solution phases 11. High Temp. Sci. 26, 313 (1989).

18. B.A. Shakhmatkin, N.M. Vedishcheva, and A.C. Wright: Can thermodynamics relate the properties of melts and glasses to their structure? J. Non-Cryst. Solids 293, 220 (2001).

19. B.A. Shakhmatkin, N.M. Vedishcheva, M.M. Shultz, and A.C. Wright: The thermodynamic properties of oxide glasses and glass-forming liquids and their chemical-structure. J. Non-Cryst. Solids 177, 249 (1994).

20. E.F. Osborn and A. Muan: Fig. 501, in Phase Diagrams for Ceramists, Vol. 1, edited by E.M. Levin, C.R. Robbins, and H.F. McMurdie, (The American Ceramic Society, Columbus, OH, 1964), p. 181.

21. G.W. Morey and N.L. Bowen: J. Soc. Glass Technol., 9, p. 232 (1925), reprinted as Fig. 482 in Phase Diagrams for Ceramists, Vol. 1, edited by E.M. Levin, C.R. Robbins, and H.F. McMurdie (The American Ceramic Society, Columbus, OH, 1964), p. 175.

22. E.F. Osborn and A. Muan: Fig. 630, in Phase Diagrams for Ceramists, Vol. 1, edited by E.M. Levin, C.R. Robbins, and H.F. McMurdie (The American Ceramic Society, Columbus, OH, 1964), p. 219.

23. ASTM C829-81: Standard practices for measurement of liquidus temperature of glass by the gradient furnace method. (re-approved 
1995), in 1999 Annual Book of ASTM Standards, Vol. 15.02 (American Society for Testing and Materials, West Conshohocken, PA, 1999).

24. R. Backman, K.H. Karlsson, M. Cable, and N.P. Pennington: Model for liquidus temperature of multi-component silicate glasses. Phys. Chem. Glasses 38, 103 (1997).

25. C. Dreyfus and G. Dreyfus: A machine learning approach to the estimation of the liquidus temperature of glass-forming oxide blends. J. Non-Cryst. Solids 318, 63 (2003).

26. H. Li, B. Jones, P. Hrma, and J.D. Vienna: Compositional effects on liquidus temperature of Hanford simulated high-level waste glasses precipitating nepheline $\left(\mathrm{NaAlSiO}_{4}\right)$. Ceram. Trans. 87, 279 (1998).

27. ASTM E1508-98: Standard guide for quantitative analysis by energy-dispersive spectroscopy, in 2003 Annual Book of ASTM Standards, Vol. 03.01 (American Society for Testing and Materials, West Conshohocken, PA, 2003).

28. E.B. Pretorius and A. Muan: Stability of $\mathrm{CaNiSi}_{2} \mathrm{O}_{6}$ ("niopside") and activity-composition relations of $\mathrm{CaMgSi}_{2} \mathrm{O}_{6}$ solid solutions at $1350^{\circ}$ C. J. Am. Ceram. Soc. 75, 1458 (1992). 